Article

\title{
Suitable DNA Barcoding for Identification and Supervision of Piper kadsura in Chinese Medicine Markets
}

\author{
Ning Yu ${ }^{1,2, \dagger}$, Hong Gu ${ }^{1,2, \dagger}$, Yulong Wei ${ }^{1,2, \dagger}$, Ning Zhu ${ }^{1,2}$, Yanli Wang ${ }^{1,2}$, Haiping Zhang ${ }^{1,2}$, \\ Yue Zhu ${ }^{1,2}$, Xin Zhang ${ }^{1,2}$, Chao Ma ${ }^{1,2, *}$ and Aidong Sun ${ }^{1,2, *}$ \\ 1 College of Biological Sciences and Technology, Beijing Forestry University, Beijing 100083, China; \\ yuning56@bjfu.edu.cn (N.Y.); guhongh9@163.com (H.G.); weiyulong1989@126.com (Y.W.); \\ ningxin1127@126.com (N.Z.); wangyanli@bjfu.edu.cn (Y.W.); z624571440@163.com (H.Z.); \\ zhuyue1109@163.com (Y.Z.); someone123667@sohu.com (X.Z.) \\ 2 Beijing Key Laboratory of Forest Food Processing and Safety, Beijing Forestry University, \\ Beijing 100083, China \\ * $\quad$ Correspondence: machao@bjfu.edu.cn (C.M.); adsun@bjfu.edu.cn (A.S.); Tel.: +86-010-6233-6700 (A.S.) \\ + These authors contributed equally to this work.
}

Academic Editor: Derek J. McPhee

Received: 20 July 2016; Accepted: 7 September 2016; Published: 12 September 2016

\begin{abstract}
Piper kadsura is a vine-like medicinal plant which is widely used in clinical treatment. However, P. kadsura is often substituted by other materials in the markets, thereby causing health risks. In this study, 38 P. kadsura samples and eight sequences from GenBank, including a closely-related species and common adulterants were collected. This study aimed to identify an effective DNA barcode from four popular DNA loci for P. kadsura authentication. The success rates of PCR amplification, sequencing, and sequence acquisition of $m a t K$ were $10.5 \%, 75 \%$, and $7.9 \%$, respectively; for $r b c L$ they were $89.5 \%, 8.8 \%$, and $7.9 \%$, respectively; ITS2 rates were $86.8 \%, 3.0 \%$, and $2.6 \%$, respectively, while for $p s b$-trnH they were all $100 \%$, which is much higher than for the other three loci. The sequences were aligned using Muscle, genetic distances were computed using MEGA 5.2.2, and barcoding gap was performed using TAXON DNA. Phylogenetic analysis showed that $p s b$-trnH could clearly distinguish $P$. kadsura from its closely related species and the common adulterant. psbA-trnH was then used to evaluate the fake proportions of P. kadsura. Results showed that $18.4 \%$ of $P$. kadsura samples were fake, indicating that adulterant species exist in the Chinese markets. Two-dimensional DNA barcoding imaging of $P$. kadsura was conducted, which was beneficial to the management of $P$. kadsura. We conclude that the $p s b A$-trnH region is a powerful tool for $P$. kadsura identification and supervision in the current medicine markets.
\end{abstract}

Keywords: Piper kadsura; DNA barcoding; $p s b A$-trnH; medicine market supervision

\section{Introduction}

Piper kadsura (Choisy) Ohwi is a vine-like medicinal plant found mostly in the Fujian, Zhejiang, and Guangdong provinces of China. The stem part of P. kadsura is a traditional medicine in China known as "haifengteng", and is harvested in summer or autumn. According to Chinese medicinal theory, P. kadsura is generally used to dredge meridian, expel wind-dampness, and relieve limb pain [1]. It is also used for cooking and improving digestive function in Japan, because its fruit is similar to pepper. To date, various constituents have been isolated from P. kadsura, including amides, lignans, terpenes, and cyclohexanes, which possess anti-human hepatitis B virus, anti-platelet activating factor, anti-insect feeding, and anti-inflammatory activities [2,3]. P. kadsura has been widely used in medical treatment and has attracted considerable attention because of its many functions. 
P. kadsura as sold in medicine markets is always dried or sliced, which makes the identification of its traditional morphological characteristics difficult. Given this, it is frequently replaced by other herbs in clinical treatment, causing potential medical problems, thereby eroding consumers' confidence. Several closely related species and other adulterants of $P$. kadsura are usually adopted as alternative herbs in many situations. Piper wallichii-a closely related species of $P$. kadsura that has no anti-inflammatory action - is often used as a substitute for P. kadsura in Zhejiang, Fujian, and Hunan provinces. Both species have the same name and are obtained in the same location. P. kadsura is commonly substituted by Kadsura heteroclite in Guangxi and Guangdong provinces, which exhibits different healing effects. The application of alternatives, to a certain extent, influences the curative effect. In extreme cases, this behavior may lead to major medical accidents. Therefore, the correct identification of $P$. kadsura is especially critical, and a practical and effective method is urgently needed.

DNA barcoding makes use of short but specific DNA tags or "barcodes" - parts of genes present in all living things - to distinguish one species from another [4]. "Barcode" is a term first coined by Hebert [5]. Many DNA barcodes have been searched. Among them are four loci-namely, matK, $r b c L$, ITS2, and $p s b A-t r n H$ - which are the most well-known in the identification of medicinal plants. The matK gene of 1084 plant species was studied, and it could be used as a standard DNA barcode for flowering plants [6]. Chase et al. concluded that $r b c L$ sequence variation is appropriate for phylogenetic analysis at the taxonomic level of seed plants [7]. Chen et al. concluded that ITS2 could be a good DNA barcode for the authentication of medicinal plants and their closely related species. They also demonstrated that $p s b A-t r n H$ is excellent for species identification [8]. Combination with the above loci for use in species authentication has been widely investigated [9-13]. In this study, we utilized DNA barcoding to discriminate $P$. kadsura species from its closely related species and common adulterants. This technique demonstrated that $p s b A-t r n H$ is the best sequence for $P$. kadsura identification.

DNA barcoding has been increasingly used for supervision of the medicine market [14-16]. Chen et al. established a barcode system, called traditional Chinese Medicine Database, based on a combination of the ITS2 and $p s b A$-trnH barcodes [17]. The system contains 78,847 sequences belonging to 23,262 medicinal species, and covers more than $95 \%$ of the herbs in pharmacopeia, including those of China, Japan, Korea, India, the USA, and Europe. The sequences provide consumer protection and safety. In the present study, we used $p s b A$-trnH to supervise commercial $P$. kadsura products in the current markets. Results indicated that adulterants are present, and that $p s b$-trnH is a powerful identification tool for P. kadsura. Portable equipment based on DNA barcoding technology is desired in the future.

\section{Results}

\subsection{Success Rates of PCR Amplification, Sequencing, and Sequence Acquisition}

Unsuccessful sequences require repeat experiments or PCR amplification reconstruction to ensure data reliability. The success rate of PCR amplification refers to the rate of samples with evident DNA straps in all the text samples. The success rate of sequencing is the rate of samples with high-quality sequences in all sequences. Sequence acquisition rate is the product of the success rate of PCR amplification and the success rate of sequencing. As shown in Figure 1, all three factors of four loci were investigated. Among the 38 samples, only four samples could be amplified with the matK locus. The amplification rates of $r b c L$ and ITS2 were $89.5 \%$ and $86.8 \%$, respectively; however, the success rates of sequencing were $8.8 \%$ and $3.0 \%$, respectively. We attempted to perform repeat experiments for the two loci, but the results remained unsatisfactory. Thus, the sequence acquisition rate of ITS2 was $2.6 \%$; mat $K$ and $r b c L, 7.9 \%$; and $p s b A$ - $t r n H, 100 \%$. Given that the sequence acquisition rate of $p s b A-t r n H$ was much greater than ITS2, matK, and $r b c L$, $p s b A-t r n H$ was chosen as the DNA barcode for P. kadsura. 


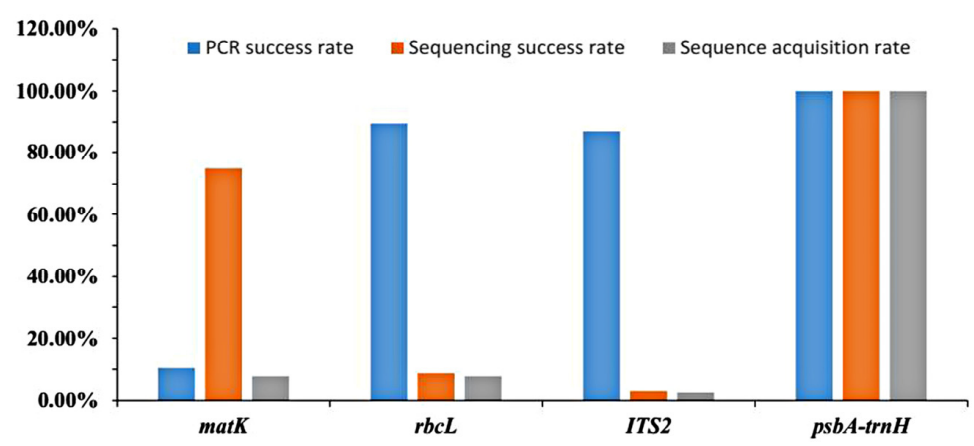

Figure 1. The success rates of PCR amplification, sequencing, and sequence acquisition.

\subsection{Genetic Distances within and between Species}

Basic Local Alignment Search Tool (BLAST) analyses were performed on the 38 sequences. The results indicated that HF007AG07, HF009AG09, HF021YZ01, HF022YZ02, HF023YZ03, HF028YZ08, and HF038BZ05 were not P. kadsura samples, but were the same adulterant (i.e., Actinidia chinensis), and we treated these samples as AC. The kimura-2-parameter genetic distances of all 46 sequences were calculated. The intraspecific distances of $P$. kadsura was 0.000 . The interspecific distance between $P$. kadsura and its adulterants varied from 0.004 to 0.426 . The minimum interspecific distance of 0.004 between $P$. kadsura and Piper wallichii was larger than the maximum intraspecific distance, proving that $p s b A-\operatorname{trn} H$ is a useful identification tool (Table 1).

Table 1. Genetic distances within and between species.

\begin{tabular}{cc}
\hline K2P Genetic Distances & Genetic Distance \\
\hline Intra-specific distances & 0.000 \\
Inter-specific distances with Piper wallichii & 0.004 \\
Inter-specific distances with AC (Actinidia chinensis) & 0.318 \\
Inter-specific distances with Kadsura heteroclita & 0.426 \\
\hline
\end{tabular}

\subsection{Barcoding Gap Assessment}

Barcoding gap is an important index to determine whether or not a DNA barcode is suitable. Barcodes should exhibit a "barcoding gap" between intra and interspecific divergences [6]. The divergence distributions were classed in 0.002 distance units to evaluate whether or not the gap exists. With $p s b A$-trnH in the P. kadsura and its adulterants matrix, there were no overlaps, and the distributions of intra and interspecific divergence were well separated, forming an evident gap, which is a positive proof for $p s b A$-trnH identification ability (Figure 2).

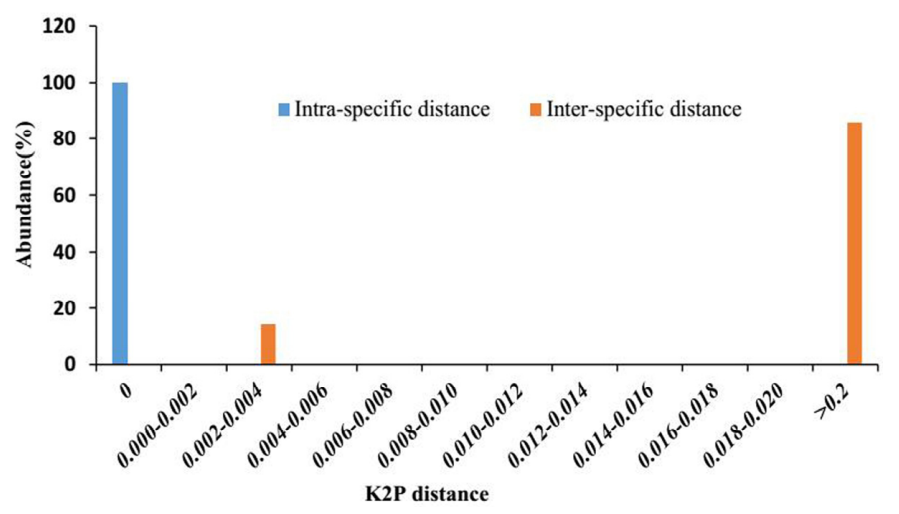

Figure 2. Relative distribution of interspecific divergence between congeneric species and intraspecific distances for $p s b A$-trnH locus. K2P: kimura-2-parameter. 


\subsection{Neighbor-Joining (NJ) Tree Identification}

An NJ tree was constructed using $46 p s b A-t r n H$ sequences (Figure 3). A total of 31 P. kadsura sequences clustered into one clade with the P. kadsura sequence downloaded from GenBank, whereas its adulterants clustered into other clades. Surprisingly, adulterants were neither $P$. wallichii nor K. heteroclite. Seven samples from Hebei Anguo (AG), Henan Yuzhou (YZ), and Anhui Bozhou (BZ) were identified as Actinidia chinensis stem. In addition to common adulterants, other fake samples that could not be easily identified by morphology were also present. Thus, DNA barcoding is necessary for authentication. We conclude that the NJ tree could effectively identify $P$. kadsura from its adulterants, including its closely related species $P$. wallichii, which further indicates that $p s b A-\operatorname{trn} H$ is a suitable DNA barcode for $P$. kadsura identification.

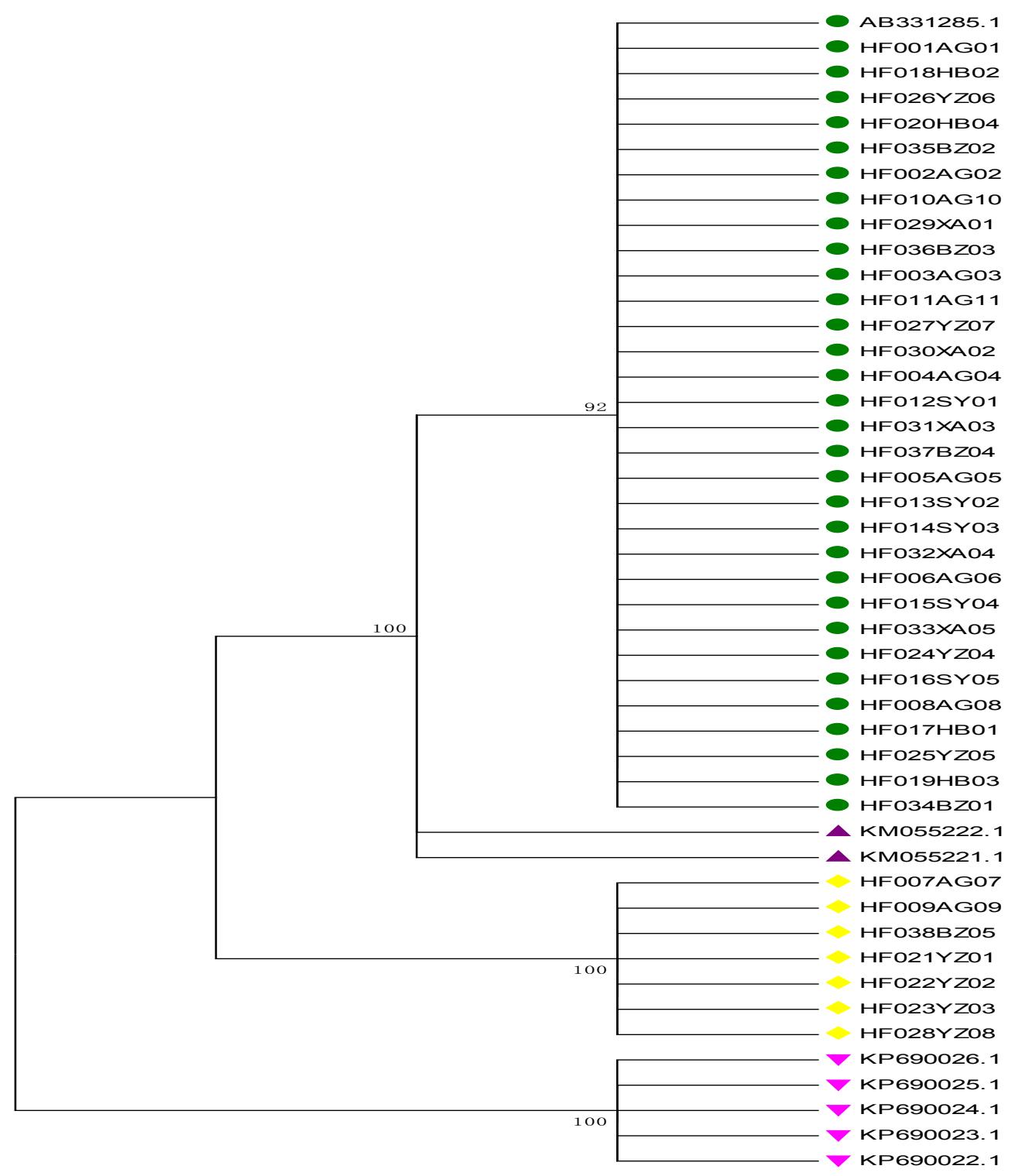

Figure 3. Phylogenetic analysis of $p s b A-t r n H$ regions for P. kadsura and its adulterants.

\subsection{Proportions of Adulterant Species}

Product adulteration and ingredient substitution are common as species of a lower market price are used to replace those of a higher price. The frequency of product mislabeling in herbal markets is estimated at $14 \%$ to $33 \%$ [18]. In this study, 38 psbA-trnH sequences were searched to 
evaluate adulterant proportions of $P$. kadsura in the current markets. The analysis of psbA-trnH region identification showed that $18.4 \%$ of samples were different from their commercial names. No fake P. kadsura samples were found in Hunan Shaoyang (SY), Heilongjiang Haerbin (HB), or Shanxi Xian (XA). The fake sample rates for AG and BZ were $18.2 \%$ and $20 \%$, respectively, whereas YZ obtained the highest rate at approximately $50 \%$ (Figure 4). The existence of adulterants poses health risks and seriously delays the development of traditional medicine.

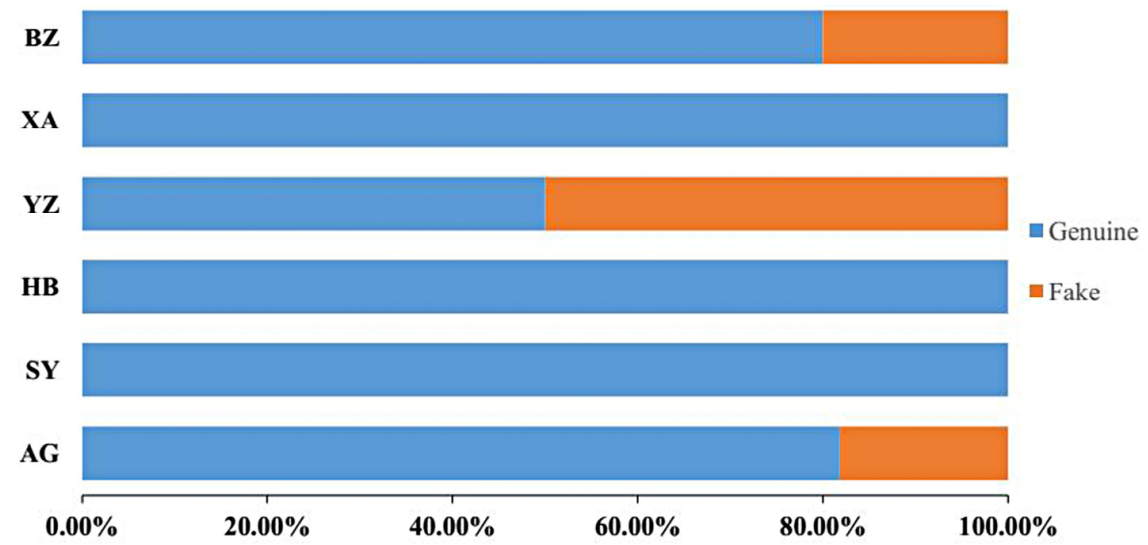

Figure 4. The adulterant rate observed for 38 P. kadsura samples.

\subsection{DNA Barcoding and Two-Dimensional DNA Barcoding Image}

DNA barcoding technology aims to identify species, similar to the barcoding of goods in the supermarket. Based on the written code and open-source PHP QR Code coding method [19], the $p s b A$-trnH sequences of P. kadsura were converted into two-dimensional DNA barcoding images (Figure 5). Two-dimensional DNA barcoding is the combination of two-dimensional technique and DNA barcoding, which is beneficial for the information conversion of DNA barcoding. Different colors represent different nucleotides, and numbers represent the length of the sequence. The sequence of P. kadsura can be obtained by scanning the two-dimensional code of the mobile terminal and then sending this sequence to a DNA barcoding database for identification, which makes identification more convenient and quick and facilitates the management of $P$. kadsura. This method can be applied to the identification of other medicinal herbs, to provide a new technical means for the protection of clinical safety.
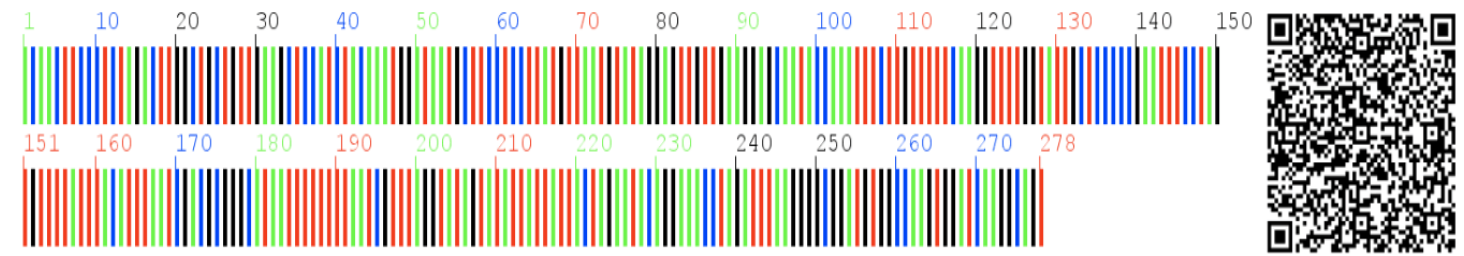

Figure 5. DNA barcoding and two-dimensional DNA barcoding image of $p s b A$-trnH sequences for P. kadsura ( $\square \mathrm{A} \square \mathrm{T} \square \mathbf{C} \square \mathbf{G})$.

\section{Discussion}

\subsection{Efficacy of psbA-trnH for Identification}

The non-coding $p s b A$-trnH spacer is the most variable plastid region in angiosperm, and it remains the leading candidate for plant DNA barcoding. The $p s b A$-trnH spacer satisfies the criteria deemed appropriate for a plant barcode, including short length (often $<500 \mathrm{bp}$ ) that allows for easy DNA extraction and amplification, high interspecific variability and divergence, and universal flanking 
primers [20-22]. The $p s b A-t r n H$ intergenic spacer region could be used as a barcode to distinguish various Dendrobium species and differentiate Dendrobium species from other adulterating species [23]. Kress et al. indicated that the rate of PCR success of $p s b A-t r n H$ with standard primers is $95.8 \%$ (46 of 48 genera), which is the highest in the nine loci searched [9]. The $p s b A$-trnH region ranked first in divergence value in six of the eight genera, and in 11 of the 14 species pairs, compared with the other eight plastid regions [24]. Moreover, $p s b A-\operatorname{trnH}$ alone showed the highest rate of identification (90\%), much higher than $r b c L a$ and matK [25]. Ma et al. concluded that $p s b A-t r n H$ is a powerful DNA marker for medicinal Pteridophytes identification [26]. In the present study, the psbA-trnH region showed the highest success rates of PCR amplification and sequencing, as well as sequence acquisition rate in P. kadsura, which was far higher than the other three loci. The inter-specific divergence of $p s b A-\operatorname{trn} H$ was much greater than intra-specific, creating an evident barcoding gap. In addition, the $p s b A$-trnH sequence could clearly identify P. kadsura from its adulterants, including closely related species, according to NJ tree description. The two-dimensional DNA barcoding image of $p s b A$-trnH sequences for $P$. kadsura also verify the importance of DNA barcoding. Our research demonstrated that $p s b A-t r n H$ is a useful tool for the identification of P. kadsura.

\subsection{Opportunities and Challenges of DNA Barcoding in Medicine Markets}

DNA barcoding is a novel system designed to provide rapid, accurate, and automated species identification by using short, standardized gene regions as internal species tags [27]. In recent years, DNA barcoding has played an increasingly significant role in the identification of Chinese medicines. Several studies were conducted to apply DNA barcoding for supervision in medicine markets. Han et al. used barcoding to investigate the proportions and varieties of adulterant species in traditional Chinese medicine (TCM) markets. A total of 1436 samples representing 295 medicinal species from seven primary TCM markets were supervised. The results showed that of the successfully generated samples, approximately $4.2 \%$ were adulterants. They suggest that a DNA barcode platform should be established for TCM market investigation [28]. Zhao et al. indicated that the ITS2 barcode could effectively identify Acanthopanacis cortex, and DNA barcoding is a convenient tool for medicine market investigation [29]. In the current study, $18.4 \%$ of $P$. kadsura samples were not genuine. Uncommon adulterants, such as $A$. chinensis, also existed. Three of the six markets were identified with fake medical materials. Certainly, $p s b A-t r n H$ is a favorable control monitor for P. kadsura quality. Thus, the current TCM markets should be regulated through a DNA barcoding method, considering the consumer's trust and clinical safety.

However, many aspects were considered in the determination of medicine quality. For instance, in the pharmacopoeia of China, medicinal effective ingredients are an important index. Whether the herbs that were identified as genuine using DNA barcoding meet the qualification of medicinal effective ingredients is also a significant topic. In addition, morphological methods still play a fundamental role in medicine identification. Thus, morphological and chemical information is necessary to integrate DNA barcoding and achieve maximum efficiency for medicinal material identification [28].

\section{Experimental Section}

\subsection{Plant Materials}

Many medical materials are dried in medicine markets. To determine the efficacy of DNA barcoding identification, we collected 38 dried $P$. kadsura samples from six approved national herbal medicine markets from six provinces in China, including 11 samples from Hebei Anguo (AG), 5 samples from Hunan Shaoyang (SY), 4 samples from Heilongiiang Haerbin (HB), 8 samples from Henan Yuzhou (YZ), 5 samples from Shanxi Xian (XA), and 5 samples from Anhui Bozhou (BZ) (Table 2), which could reflect $P$. kadsura quality condition in the current market. All 38 specimens were deposited in Beijing Forestry University. One P. kadsura sequence, two P. wallichii sequences, and five K. heteroclite sequences were also downloaded from GenBank (Table 3). 
Table 2. The list of 38 samples used in this study. AG: Hebei Anguo; BZ: Anhui Bozhou; HB: Heilongjiang Haerbin; SY: Hunan Shaoyang; XA: Shanxi Xian; YZ: Henan Yuzhou.

\begin{tabular}{|c|c|c|c|c|}
\hline S1 No. & Latin Name & Place of Collection & Sample No. & Identification Result \\
\hline 1 & P. kadsura & AG & HF001AG01 & Genuine \\
\hline 2 & P. kadsura & AG & HF002AG02 & Genuine \\
\hline 3 & P. kadsura & AG & HF003AG03 & Genuine \\
\hline 4 & P. kadsura & AG & HF004AG04 & Genuine \\
\hline 5 & P. kadsura & AG & HF005AG05 & Genuine \\
\hline 6 & P. kadsura & AG & HF006AG06 & Genuine \\
\hline 7 & P. kadsura & AG & HF007AG07 & Fake \\
\hline 8 & P. kadsura & AG & HF008AG08 & Genuine \\
\hline 9 & P. kadsura & AG & HF009AG09 & Fake \\
\hline 10 & P. kadsura & AG & HF010AG10 & Genuine \\
\hline 11 & P. kadsura & AG & HF011AG11 & Genuine \\
\hline 12 & P. kadsura & SY & HF012SY01 & Genuine \\
\hline 13 & P. kadsura & SY & HF013SY02 & Genuine \\
\hline 14 & P. kadsura & SY & HF014SY03 & Genuine \\
\hline 15 & P. kadsura & SY & HF015SY04 & Genuine \\
\hline 16 & P. kadsura & SY & HF016SY05 & Genuine \\
\hline 17 & P. kadsura & HB & HF017HB01 & Genuine \\
\hline 18 & P. kadsura & HB & HF018HB02 & Genuine \\
\hline 19 & P. kadsura & HB & HF019HB03 & Genuine \\
\hline 20 & P. kadsura & HB & HF020HB04 & Genuine \\
\hline 21 & P. kadsura & $Y Z$ & HF021YZ01 & Fake \\
\hline 22 & P. kadsura & $Y Z$ & HF022YZ02 & Fake \\
\hline 23 & P. kadsura & $Y Z$ & HF023YZ03 & Fake \\
\hline 24 & P. kadsura & $Y Z$ & HF024YZ04 & Genuine \\
\hline 25 & P. kadsura & $Y Z$ & HF025YZ05 & Genuine \\
\hline 26 & P. kadsura & $Y Z$ & HF026YZ06 & Genuine \\
\hline 27 & P. kadsura & $\mathrm{YZ}$ & HF027YZ07 & Genuine \\
\hline 28 & P. kadsura & $\mathrm{YZ}$ & HF028YZ08 & Fake \\
\hline 29 & P. kadsura & XA & HF029XA01 & Genuine \\
\hline 30 & P. kadsura & XA & HF030XA02 & Genuine \\
\hline 31 & P. kadsura & XA & HF031XA03 & Genuine \\
\hline 32 & P. kadsura & XA & HF032XA04 & Genuine \\
\hline 33 & P. kadsura & XA & HF033XA05 & Genuine \\
\hline 34 & P. kadsura & $\mathrm{BZ}$ & HF034BZ01 & Genuine \\
\hline 35 & P. kadsura & $\mathrm{BZ}$ & HF035BZ02 & Genuine \\
\hline 36 & P. kadsura & $\mathrm{BZ}$ & HF036BZ03 & Genuine \\
\hline 37 & P. kadsura & $\mathrm{BZ}$ & HF037BZ04 & Genuine \\
\hline 38 & P. kadsura & $\mathrm{BZ}$ & HF038BZ05 & Fake \\
\hline
\end{tabular}

Table 3. The list of accessions downloaded from GenBank.

\begin{tabular}{cccc}
\hline No. & Latin Name & Genus Name & Genebank No. \\
\hline 1 & P. kadsura & Piper Linn. & AB331285.1 \\
2 & P. kadsura & Piper Linn. & KM055222.1 \\
3 & P. wallichii & Piper Linn. & KM055221.1 \\
4 & K. heteroclita & Kadsura Kaempf. ex Juss. & KP690026.1 \\
5 & K. heteroclita & Kadsura Kaempf. ex Juss. & KP690025.1 \\
6 & K. heteroclita & Kadsura Kaempf. ex Juss. & KP690024.1 \\
7 & K. heteroclita & Kadsura Kaempf. ex Juss. & KP690023.1 \\
8 & K. heteroclita & Kadsura Kaempf. ex Juss. & KP690022.1 \\
\hline
\end{tabular}

\subsection{DNA Extraction, Amplification, and Sequencing}

The samples were first scraped and then wiped with 75\% ethanol to prevent fungal contamination. Total DNA extraction was achieved using a plant genomic DNA kit (Tiangen, Beijing, China), 
based on the Hexadecyltrimethy Ammonium Bromide (CTAB) approach. The primers used for all four regions and the PCR conditions were as follows: ITS2: S2F, ATGCGATACTTGGTGTGAAT; S3R, GACGCTTCTCCAGACTACAAT; PCR conditions: $94{ }^{\circ} \mathrm{C} 5 \mathrm{~min}, 40$ cycles at $94{ }^{\circ} \mathrm{C} 30 \mathrm{~s}, 56{ }^{\circ} \mathrm{C}$ $30 \mathrm{~s}, 72{ }^{\circ} \mathrm{C} 45 \mathrm{~s}$, and $72{ }^{\circ} \mathrm{C} 10 \mathrm{~min} . \quad p s b A-t r n H:$ fwd. PA, GTTATGCATGAACGTAATGCTC; rev. TH, CGCGCATGGTGGATTCACAATCC; PCR conditions: $94{ }^{\circ} \mathrm{C} 5 \mathrm{~min}, 30$ cycles at $94{ }^{\circ} \mathrm{C}$ $1 \mathrm{~min}, 55^{\circ} \mathrm{C} 1 \mathrm{~min}, 72{ }^{\circ} \mathrm{C} 1.5 \mathrm{~min}$, and $72{ }^{\circ} \mathrm{C} 10 \mathrm{~min}$. mat $\mathrm{K}: 390 \mathrm{~F}, \mathrm{CGATCTATTCATTCAATATTTC}$; 1326R, TCTAGCACACGAAAGTCGAAGT; PCR conditions: $94{ }^{\circ} \mathrm{C} 5 \mathrm{~min}$, 30 cycles at $94{ }^{\circ} \mathrm{C}$ $1 \mathrm{~min}, 48{ }^{\circ} \mathrm{C} 30 \mathrm{~s}, 72{ }^{\circ} \mathrm{C} 1 \mathrm{~min}$, and $72{ }^{\circ} \mathrm{C} 10 \mathrm{~min} . \quad r b c L: 1 \mathrm{f}$, ATGTCACCACAAACAGAAAC; 724r, TCGCATGTACCTGCAGTAGC; PCR conditions: $95^{\circ} \mathrm{C} 2 \mathrm{~min}, 34$ cycles at $94{ }^{\circ} \mathrm{C} 1 \mathrm{~min}, 55^{\circ} \mathrm{C} 30 \mathrm{~s}$, $72{ }^{\circ} \mathrm{C} 1 \mathrm{~min}$, and $72{ }^{\circ} \mathrm{C} 10 \mathrm{~min}[8]$.

\subsection{Sequence Alignment and Genetic and Phylogenetic Analyses}

CodonCode Aligner 4.2.7 (CodonCode Co., Centerville, MA, USA) was used to trim and assemble raw trace files. BLAST analysis was performed using the nucleotide database at National Center for Biotechnology Information (NCBI). Intra- and inter-species genetic distances were obtained with the kimura-2-parameter (K2P) model of MEGA 5.2.2 [30]. Intra- and inter-species pairwise divergences were calculated as a barcoding gap by using TAXON DNA [31]. A neighbor-joining (NJ) tree based on phylogenetic analysis was constructed, with 1000 replicate bootstrap tests to identify $P$. kadsura via MEGA 5.2.2 [30].

Acknowledgments: This work was supported by Forestry public welfare industry research special funds (201504606).

Author Contributions: Aidong Sun and Chao Ma conceived and designed research; Ning Yu, Hong Gu, Yulong Wei, Ning Zhu, Yanli Wang, Haiping Zhang, Yue Zhu and Xin Zhang conducted experiments; Ning $\mathrm{Yu}$, Hong Gu and Yulong Wei analyzed data; Ning Yu and Yulong Wei wrote the paper; Aidong Sun and Chao Ma critically reviewed the manuscript. Collectively the group is interested in investigating evolutionary process and plant speciation.

Conflicts of Interest: The authors declare no conflict of interest.

\section{Abbreviations}

The following abbreviations are used in this manuscript:

TCM

$\mathrm{K} 2 \mathrm{P}$

Traditional Chinese medicine

NJ Kimura 2-Parameter

Neighbor-joining

\section{References}

1. State Pharmacopoeia Committee. Chinese Pharmacopoeia, 10th ed.; Medical Science and Technology Press: Beijing, China, 2010; p. 448.

2. Kuo, Y.C.; Yang, N.S.; Chou, C.J.; Lin, L.C.; Tsai, W.J. Regulation of cell proliferation, gene expression, production of cytokines, and cell cycle progression in primary human $\mathrm{T}$ lymphocytes by piperlactam $\mathrm{S}$ isolated from Piper kadsura. Mol. Pharmacol. 2000, 58, 1057-1066. [PubMed]

3. Kim, K.H.; Choi, J.W.; Choi, S.U.; Ha, S.K.; Kim, S.Y.; Park, H.J.; Lee, K.R. The chemical constituents of Piper kadsura and their cytotoxic and anti-neuroinflammtaory activities. J. Enzyme Inhib. Med. Chem. 2011, 26, 254-260. [CrossRef] [PubMed]

4. Marshall, E. Will DNA bar codes breathe life into classification? Science 2005, 307, 1037. [CrossRef] [PubMed]

5. Hebert, P.D.N.; Cywinska, A.; Ball, S.L.; DeWaard, J.R. Biological identifications through DNA barcodes. Proc. R. Soc. Lond. B Biol. Sci. 2003, 270, 313-321. [CrossRef] [PubMed]

6. Lahaye, R.; van der Bank, M.; Bogarin, D.; Warner, J.; Pupulin, F.; Gigot, G.; Maurin, O.; Duthoit, S.; Barraclough, T.G.; Savolainen, V. DNA barcoding the floras of biodiversity hotspots. Proc. Natl. Acad. Sci. USA 2008, 105, 2923-2928. [CrossRef] [PubMed] 
7. Chase, M.W.; Soltis, D.E.; Olmstead, R.G.; Morgan, D.; Les, D.H.; Mishler, B.D.; Duvall, M.R.; Price, R.A.; Hills, H.G.; Qiu, Y.L.; et al. Phylogenetics of seed plants: An analysis of nucleotide sequences from the plastid gene rbcL. Ann. Mo. Bot. Gard. 1993, 80, 528-580. [CrossRef]

8. Chen, S.L.; Yao, H.; Han, J.P.; Liu, C.; Song, J.Y.; Shi, L.C.; Zhu, Y.J.; Ma, X.Y.; Gao, T.; Pang, X.H.; et al. Validation of the ITS2 region as a novel DNA barcode for identifying medicinal plant species. PLoS ONE 2010, 5, e8613. [CrossRef] [PubMed]

9. Kress, W.J.; Erickson, D.L. A two-locus global DNA barcode for land plants: The coding rbcL gene complements the non-coding trnH-psbA spacer region. PLoS ONE 2007, 2, e508. [CrossRef] [PubMed]

10. Newmaster, S.G.; Fazekas, A.J.; Steeves, R.A.D.; Janovec, J. Testing candidate plant barcode regions in the Myristicaceae. Mol. Ecol. Resour. 2008, 8, 480-490. [CrossRef] [PubMed]

11. CBOL Plant Working Group. A DNA barcode for land plants. Proc. Natl. Acad. Sci. USA 2009, 106, $1294-1297$.

12. Hou, D.Y.; Song, J.Y.; Shi, L.C.; Ma, X.C.; Xin, T.Y.; Han, J.P.; Xiao, W.; Sun, Z.Y.; Cheng, R.Y.; Yao, H. Stability and accuracy assessment of identification of traditional Chinese materia medica using DNA barcoding: A case study on Flos Lonicerae Japonicae. BioMed Res. Int. 2013, 2013, 1-6. [CrossRef] [PubMed]

13. Vassou, S.L.; Kusuma, G.; Parani, M. DNA barcoding for species identification from dried and powdered plant parts: A case study with authentication of the raw drug market samples of Sida cordifolia. Gene 2015, 559, 86-93. [CrossRef] [PubMed]

14. Kool, A.; de Boer, H.J.; Krüger, Å.; Rydberg, A.; Abbad, A.; Björk, L.; Martin, G. Molecular identification of commercialized medicinal plants in Southern Morocco. PLoS ONE 2012, 7, e39459. [CrossRef] [PubMed]

15. Xin, T.Y.; Li, X.J.; Yao, H.; Lin, Y.L.; Ma, X.C.; Cheng, R.Y.; Song, J.Y.; Ni, L.H.; Fan, C.Z.; Chen, S.L. Survey of commercial Rhodiola products revealed species diversity and potential safety issues. Sci. Rep. 2015, 5, 8337. [CrossRef] [PubMed]

16. Wu, L.; Sun, W.; Wang, B.; Zhao, H.Y.; Li, Y.L.; Cai, S.Q.; Xiang, L.; Zhu, Y.J.; Yao, H.; Song, J.Y.; et al. An integrated system for identifying the hidden assassins in traditional medicines containing aristolochic acids. Sci. Rep. 2015, 5, 11318. [CrossRef] [PubMed]

17. Chen, S.L.; Pang, X.; Song, J.; Shi, L.; Yao, H.; Han, J.; Leon, C. A renaissance in herbal medicine identification: From morphology to DNA. Biotechnol. Adv. 2014, 32, 1237-1244. [CrossRef] [PubMed]

18. Newmaster, S.G.; Grguric, M.; Shanmughanandhan, D.; Ramalingam, S.; Ragupathy, S. DNA barcoding detects contamination and substitution in North American herbal products. BMC Med. 2013, 11, 222. [CrossRef] [PubMed]

19. Chen, S.L. Standard DNA Barcodes of Chinese Material Medica in Chinese Pharmacopoeia; Science Press: Beijing, China, 2015; p. 18.

20. Shaw, J.; Lickey, E.B.; Beck, J.T.; Farmer, S.B.; Liu, W.S.; Miller, J.; Siripun, K.C.; Winder, C.T.; Schilling, E.E.; Small, R.L. The tortoise and the hare II: Relative utility of 21 noncoding chloroplast DNA sequences for phylogenetic analysis. Am. J. Bot. 2005, 92, 142-166. [CrossRef] [PubMed]

21. Whitlock, B.A.; Hale, A.M.; Groff, P.A. Intraspecific inversions pose a challenge for the trnH-psbA plant DNA barcode. PLoS ONE 2010, 5, e11533. [CrossRef] [PubMed]

22. Pang, X.H.; Liu, C.; Shi, L.C.; Liu, R.; Liang, D.; Li, H.; Cherny, S.S.; Chen, S.L. Utility of the trnH-psbA intergenic spacer region and its combinations as plant DNA barcodes: A meta-analysis. PLoS ONE 2012, 7, e48833. [CrossRef] [PubMed]

23. Yao, H.; Song, J.Y.; Ma, X.Y.; Liu, C.; Li, Y.; Xu, H.X.; Chen, S.L. Identification of Dendrobium species by a candidate DNA barcode sequence: The chloroplast $p s b A$-trnH intergenic region. Planta Med. 2009, 75, 667-669. [CrossRef] [PubMed]

24. Kress, W.J.; Wurdack, K.J.; Zimmer, E.A.; Weigt, L.A.; Janzen, D.H. Use of DNA barcodes to identify flowering plants. Proc. Natl. Acad. Sci. USA 2005, 102, 8369-8374. [CrossRef] [PubMed]

25. Kress, W.J.; Erickson, D.L.; Jones, F.A.; Swenson, N.G.; Perez, R.; Sanjur, O.; Bermingham, E. Plant DNA barcodes and a community phylogeny of a tropical forest dynamics plot in Panama. Proc. Natl. Acad. Sci. USA 2009, 106, 18621-18626. [CrossRef] [PubMed]

26. Ma, X.Y.; Xie, C.X.; Liu, C.; Song, J.Y.; Yao, H.; Luo, K.; Zhu, Y.J.; Gao, T.; Pang, X.H.; Chen, S.L.; et al. Species identification of medicinal pteridophytes by a DNA barcode marker, the chloroplast $p s b$-trnH intergenic region. Biol. Pharm. Bull. 2010, 33, 1919-1924. [CrossRef] [PubMed]

27. Hebert, P.D.; Gregory, T.R. The promise of DNA barcoding for taxonomy. Syst. Boil. 2005, 54, 852-859. [CrossRef] [PubMed] 
28. Han, J.P.; Pang, X.H.; Liao, B.S.; Yao, H.; Song, J.Y.; Chen, S.L. An authenticity survey of herbal medicines from markets in China using DNA barcoding. Sci. Rep. 2016, 6, 18723. [CrossRef] [PubMed]

29. Zhao, S.; Chen, X.C.; Song, J.Y.; Pang, X.H.; Chen, S.L. Internal transcribed spacer 2 barcode: A good tool for identifying Acanthopanacis cortex. Front. Plant Sci. 2015, 6, 840. [CrossRef] [PubMed]

30. Tamura, K.; Peterson, D.; Peterson, N.; Stecher, G.; Nei, M.; Kumar, S. MEGA5: Molecular evolutionary genetics analysis using maximum likelihood, evolutionary distance, and maximum parsimony methods. Mol. Biol. Evol. 2011, 28, 2731-2739. [CrossRef] [PubMed]

31. Slabbinck, B.; Dawyndt, P.; Martens, M.; de Vos, P.; de Baets, B. Taxon Gap: A visualization tool for intra-and inter-species variation among individual biomarkers. Bioinformatics 2008, 24, 866-867. [CrossRef] [PubMed]

Sample Availability: Not Available.

(C) 2016 by the authors; licensee MDPI, Basel, Switzerland. This article is an open access article distributed under the terms and conditions of the Creative Commons Attribution (CC-BY) license (http://creativecommons.org/licenses/by/4.0/). 